\title{
Equal Area Laws and Latent Heat for $d$-Dimensional RN-AdS Black Hole
}

\author{
Li-Chun Zhang, ${ }^{1,2}$ Hui-Hua Zhao, ${ }^{1,2}$ Ren Zhao, ${ }^{1,2}$ and Meng-Sen $\mathrm{Ma}^{1,2}$ \\ ${ }^{1}$ Department of Physics, Shanxi Datong University, Datong 037009, China \\ ${ }^{2}$ Institute of Theoretical Physics, Shanxi Datong University, Datong 037009, China
}

Correspondence should be addressed to Ren Zhao; zhao2969@sina.com

Received 9 August 2014; Revised 31 October 2014; Accepted 6 November 2014; Published 19 November 2014

Academic Editor: George Siopsis

Copyright (C) $2014 \mathrm{Li}$-Chun Zhang et al. This is an open access article distributed under the Creative Commons Attribution License, which permits unrestricted use, distribution, and reproduction in any medium, provided the original work is properly cited. The publication of this article was funded by SCOAP ${ }^{3}$.

We study the equal area laws of $d$-dimensional RN-AdS black hole. We choose two kinds of phase diagrams, $P$ - $V$ and $T$-S. We employ the equal area laws to find an isobar which is the real two-phase coexistence line. Our calculation is much simpler to derive the critical value of the thermodynamic quantities. According to the thermodynamic quantities, we also study the latent heat of the black hole.

\section{Introduction}

Like ordinary thermodynamic matter, black holes also have temperature, entropy, and energy. The laws of black hole mechanics have similar forms to the laws of thermodynamics [1]. Therefore, we can treat black holes as thermodynamic systems. In fact, between black holes and the conventional thermodynamic systems, there are other similarities, such as phase transition and critical behaviors. The pioneering work of Davies [2] and the well-known Hawking-Page phase transition [3] are both proposed to elaborate these points. The phase transitions and critical phenomena in anti-de Sitter (AdS) black holes have been studied extensively [4-10]. Some interesting works show that there exists phase transition similar to the Van der Waals liquid/gas phase transition for some black holes [11-21]. Even for the black holes in dS space critical behaviors can also be studied by considering the connections between the black hole horizon and the cosmological horizon $[22,23]$.

The studies on the phase transition of black holes by considering cosmological constant as a variable have got many attentions [24-28]. For AdS black holes, the cosmological constant is connected to the pressure in general thermodynamic system, the relation is

$$
P=-\frac{1}{8 \pi} \Lambda=\frac{3}{8 \pi} \frac{1}{l^{2}}
$$

and the corresponding thermodynamic volume is

$$
V=\left(\frac{\partial M}{\partial P}\right)_{S, Q_{i} J_{k}} .
$$

Theoretically, if considering black holes in AdS spacetime as a thermodynamic system, the critical behaviors and phase transitions should also exist. It has been shown that this transition is superficially analogous to the phase transition of Van der Waals (VdM) liquid-gas system. It is well known that for the VdW system above the critical temperature $T_{c}$ the isothermal curves display similar behavior to the experimental result. However, below the critical temperature $T_{c}$, in the coexistence line there will be a regime where $(\partial p / \partial V)_{T}>0$. Therefore, there, the condition of stability and equilibrium violate. Experiments show that there should be a horizontal isobar in the isotherm to represent the condensation line; here the gas coexists with the liquid. On crossing the line from the gas side the system begins to form droplets of liquid on the condensation line, which grow and coalesce until all the material has passed to the liquid phase.

According to Maxwell's equal area law, one can replace the oscillatory part with an isobar which represents the liquid-gas coexistence line. Thus the experimental result is consistent with the theoretical one. Since the AdS black hole is similar to the VdW system, in the process of phase transition of the black holes there should be similar isobar to represent 
the two-phase coexistence line. In [29], the authors have studied the equal area law in the $T-S$ plane for the charged AdS black hole. In this paper we utilize a different method to construct the equal area law in the $P-V$ plane. Specifically, our method can supply an efficient way to derive the critical thermodynamic quantities.

\section{2. $P-V$ and $T-S$ Phase Diagrams}

Reissner-Nordstrom black holes are characterized by their mass $(M)$ and charge $(Q)$. The solution for $d$-dimensional RN-AdS spacetime with a negative cosmological constant $\left(\Lambda=-(d-1)(d-2) / 2 l^{2}\right)$ is defined by the line element [29]

$$
\begin{aligned}
d s^{2} & =-f d t^{2}+f^{-1} d r^{2}+r^{2} d \Omega_{d-2}, \\
F & =d A \\
A & =-\sqrt{\frac{d-2}{2(d-3)}} \frac{q}{r^{d-3}} d t
\end{aligned}
$$

where

$$
f=1-\frac{m}{r^{d-2}}+\frac{q^{2}}{r^{2(d-3)}}+\frac{r^{2}}{l^{2}} .
$$

The ADM mass and the electric charge have been identified as

$$
\begin{aligned}
& M=\frac{(d-2) \omega_{d-2}}{16 \pi} m, \\
& Q=\frac{\sqrt{2(d-2)(d-3)} \omega_{d-2}}{8 \pi} q,
\end{aligned}
$$

where the volume of the unit $d$-sphere $\omega_{d}$ can be expressed as

$$
\omega_{d}=\frac{2 \pi^{(d+1) / 2}}{\Gamma((d+1) / 2)} .
$$

The corresponding Hawking temperature, entropy, and electric potential have been reviewed as

$$
\begin{aligned}
T= & \frac{(d-3)}{4 \pi}\left(\frac{4}{\omega_{d-2}}\right)^{-1 /(d-2)} S^{1 /(d-2)} \\
& -q^{2} \frac{(d-3)}{4 \pi}\left(\frac{4}{\omega_{d-2}}\right)^{(5-2 d) /(d-2)} S^{(5-2 d) /(d-2)} \\
& +\frac{4}{(d-2)}\left(\frac{4}{\omega_{d-2}}\right)^{1 /(d-2)} P S^{1 /(d-2)}, \\
S= & \frac{\omega_{d-2} r_{+}^{d-2}}{4}, \\
\Phi= & \sqrt{\frac{d-2}{2(d-3)}} \frac{q}{r_{+}^{d-3}} .
\end{aligned}
$$

These quantities satisfy the first law of black hole thermodynamics

$$
d M=T d S+\Phi d Q+V d P
$$

where the thermodynamic volume $V=\left(\omega_{d-2} r_{+}^{d-1}\right) /(d-1)$ and the effective pressure $P=-\Lambda /\left(8 \pi l^{2}\right)=((d-1)(d-$ 2)) $/\left(16 \pi l^{2}\right)$.

For this black hole there is the equation of state [26]

$$
P=\frac{T(d-2)}{4 r_{+}}-\frac{(d-3)(d-2)}{16 \pi r_{+}^{2}}+\frac{q^{2}(d-3)(d-2)}{16 \pi r_{+}^{2(d-2)}},
$$

from which one can depict $P-V$ curves for given temperature $T$ at constant $Q$.

When the number of particles in a system is fixed, the internal energy $U(V, T)$ is a function of state. If the temperature $T$ is given, the internal energy is only dependent on the volume $V$. Thus the energy difference between two states with volumes $V_{1}, V_{2}$ at the same $T$ is

$$
\Delta U=-\int_{V_{1}}^{V_{2}} P(V) d V
$$

Because $U$ is a function of state, the result of $\Delta U$ is independent of the path of integral. Thus, to find the equal area isobar $P=P_{0}$, one can employ the relation

$$
P_{0}\left(V_{2}-V_{1}\right)=\int_{V_{2}}^{V_{1}} P(V) d V
$$

Substituting (9) into (11), we can obtain

$$
\begin{aligned}
P_{0} r_{2}^{2(d-2)} x^{d-3} \frac{\left(1-x^{d-1}\right)}{d-1} & \\
= & \frac{T}{4} r_{2}^{2 d-5} x^{d-3}\left(1-x^{d-2}\right) \\
& -\frac{(d-2)}{16 \pi} r_{2}^{2(d-3)} x^{d-3}\left(1-x^{d-3}\right) \\
& +\frac{q^{2}(d-2)}{16 \pi}\left(1-x^{d-3}\right),
\end{aligned}
$$

where $r_{1}, r_{2}$ are the intersection points of the isobar with the isothermal curves and we set $x=r_{1} / r_{2}$; thus $0 \leq x \leq 1$. The points $r_{1}, r_{2}$ in the isothermal curves should satisfy

$$
\begin{aligned}
& P_{0}=\frac{T(d-2)}{4 r_{2}}-\frac{(d-3)(d-2)}{16 \pi r_{2}^{2}}+\frac{q^{2}(d-3)(d-2)}{16 \pi r_{2}^{2(d-2)}}, \\
& P_{0}=\frac{T(d-2)}{4 r_{1}}-\frac{(d-3)(d-2)}{16 \pi r_{1}^{2}}+\frac{q^{2}(d-3)(d-2)}{16 \pi r_{1}^{2(d-2)}} .
\end{aligned}
$$

Adding the two equations above, we can derive

$$
\begin{aligned}
2 P_{0} r_{2}^{2(d-2)} x^{2(d-2)} & \\
= & \frac{T(d-2)}{4} r_{2}^{2 d-5} x^{2 d-5}(1+x) \\
& -\frac{(d-3)(d-2)}{16 \pi} r_{2}^{2(d-3)} x^{2(d-3)}\left(1+x^{2}\right) \\
& +\frac{q^{2}(d-3)(d-2)}{16 \pi}\left(1+x^{2(d-2)}\right) .
\end{aligned}
$$


Subtracting (14) from (13), one can obtain

$$
\begin{aligned}
\operatorname{Tr}_{2}^{2 d-5} x^{2 d-5}= & \frac{(d-3)}{4 \pi} r_{2}^{2(d-3)} x^{2(d-3)}(1+x) \\
& -\frac{q^{2}(d-3)}{4 \pi} \frac{\left(1-x^{2(d-2)}\right)}{1-x} .
\end{aligned}
$$

From (12), (15), we derive

$$
\begin{aligned}
\frac{T}{2} r_{2}^{2 d-5} x^{2(d-2)} \frac{\left(1-x^{d-2}\right)}{\left(1-x^{d-1}\right)} & \\
- & \frac{(d-2)}{8 \pi} r_{2}^{2(d-3)} x^{2(d-2)} \frac{\left(1-x^{d-3}\right)}{\left(1-x^{d-1}\right)} \\
& +\frac{q^{2}(d-2)}{8 \pi} x^{d-1} \frac{\left(1-x^{d-3}\right)}{\left(1-x^{d-1}\right)} \\
= & \frac{T(d-2)}{4(d-1)} r_{2}^{2 d-5} x^{2 d-5}(1+x) \\
& -\frac{(d-3)(d-2)}{16 \pi(d-1)} r_{2}^{2(d-3)} x^{2(d-3)}\left(1+x^{2}\right) \\
& +\frac{q^{2}(d-3)(d-2)}{16 \pi(d-1)}\left(1+x^{2(d-2)}\right) .
\end{aligned}
$$

Substituting (16) into (17), we have

$$
\begin{aligned}
& r_{2}^{2(d-3)} x^{2 d-5}(d-3) \frac{(1+x)\left(1-x^{d-2}\right)}{1-x^{d-1}} \\
& -q^{2}(d-3) \frac{x\left(1-x^{d-2}\right)\left(1-x^{2(d-2)}\right)}{\left(1-x^{d-1}\right)(1-x)} \\
& -r_{2}^{2(d-3)} x^{2(d-2)}(d-2) \frac{\left(1-x^{d-3}\right)}{\left(1-x^{d-1}\right)} \\
& +q^{2} x^{d-1}(d-2) \frac{\left(1-x^{d-3}\right)}{\left(1-x^{d-1}\right)} \\
& =\frac{(d-3)(d-2)}{2(d-1)} r_{2}^{2(d-3)} x^{2(d-3)}(1+x)^{2} \\
& -\frac{q^{2}(d-3)(d-2)}{2(d-1)} \frac{(1+x)\left(1-x^{2(d-2)}\right)}{1-x} \\
& -\frac{(d-3)(d-2)}{2(d-1)} r_{2}^{2(d-3)} x^{2(d-3)}\left(1+x^{2}\right) \\
& +\frac{q^{2}(d-3)(d-2)}{2(d-1)}\left(1+x^{2(d-2)}\right) \text {. }
\end{aligned}
$$

We can simplify (18) and write it in the form

$$
r_{2}^{2(d-3)}=q^{2} \frac{y_{1}(x)}{y_{2}(x)},
$$

with

$$
\begin{aligned}
y_{2}(x)= & x^{2 d-5}(1-x) \\
\cdot & {\left[(d-3)(1+x)\left(1-x^{d-2}\right)\right.} \\
& -x(d-2)\left(1-x^{d-3}\right) \\
& \left.-\frac{(d-3)(d-2)}{(d-1)}\left(1-x^{d-1}\right)\right], \\
y_{1}(x)=- & \quad(d-3)(d-2) \\
& \cdot x(1-1) \\
+ & (d-3) x\left(1-x^{2 d-5}\right)\left(1-x^{d-1}\right) \\
& -(d-2) x^{d-1}\left(1-x^{d-3}\right)(1-x) .
\end{aligned}
$$

Substituting (19) into (16), we can obtain

$$
\begin{gathered}
T\left(q^{2} \frac{y_{1}(x)}{y_{2}(x)}\right)^{(2 d-5) /(2 d-6)} x^{2 d-5} \\
=q^{2} \frac{(d-3)}{4 \pi} x^{2(d-3)}(1+x) \frac{y_{1}(x)}{y_{2}(x)} \\
-\frac{q^{2}(d-3)}{4 \pi} \frac{\left(1-x^{2(d-2)}\right)}{1-x} .
\end{gathered}
$$

When $x=1$, there is $r_{1}=r_{2}=r_{c}$, which is the critical point. In this case, we can obtain from (19)

$$
r_{c}^{2(d-3)}=q^{2}(d-2)(2 d-5) .
$$

According to (21), when $x=1$,

$$
T_{c}=\frac{(d-3)^{2}}{\pi(2 d-5) r_{c}}
$$

(18) We set

$$
T=\chi T_{c}
$$

Equation (21) becomes

$$
\begin{gathered}
\chi \frac{(d-3)^{2}}{\pi(2 d-5)[(d-2)(2 d-5)]^{1 /(2 d-6)}} \\
\cdot\left(\frac{y_{1}(x)}{y_{2}(x)}\right)^{(2 d-5) /(2 d-6)} x^{2 d-5} \\
=q^{2} \frac{(d-3)}{4 \pi} x^{2(d-3)}(1+x) \frac{y_{1}(x)}{y_{2}(x)} \\
-\frac{q^{2}(d-3)}{4 \pi} \frac{\left(1-x^{2(d-2)}\right)}{1-x} .
\end{gathered}
$$


Because the coexisting phases have the same free energy, one can derive an equal area relation

$$
T_{0}\left(S_{2}-S_{1}\right)=\int_{S_{1}}^{S_{2}} T d S .
$$

The equation of state (9) can be changed into

$$
T=4 P \frac{r_{+}}{(d-2)}+\frac{(d-3)}{4 \pi r_{+}}-\frac{q^{2}(d-3)}{4 \pi r_{+}^{2 d-5}}
$$

Substituting (27) into (26), we obtain

$$
\begin{gathered}
T_{0} r_{2}^{2 d-5} x^{d-3}\left(1-x^{d-2}\right) \\
=\frac{4 P}{d-1} r_{2}^{2 d-4} x^{d-3}\left(1-x^{d-1}\right) \\
+\frac{(d-2)}{4 \pi} r_{2}^{2 d-6} x^{d-3}\left(1-x^{d-3}\right) \\
-\frac{q^{2}(d-2)}{4 \pi}\left(1-x^{d-3}\right) .
\end{gathered}
$$

For the two points $r_{1}, r_{2}$, there are, respectively,

$$
\begin{aligned}
& T_{0}=4 P \frac{r_{2}}{(d-2)}+\frac{(d-3)}{4 \pi r_{2}}-\frac{q^{2}(d-3)}{4 \pi r_{2}^{2 d-5}}, \\
& T_{0}=4 P \frac{r_{1}}{(d-2)}+\frac{(d-3)}{4 \pi r_{1}}-\frac{q^{2}(d-3)}{4 \pi r_{1}^{2 d-5}} .
\end{aligned}
$$

Combining (29) can give

$$
\begin{aligned}
4 P \frac{r_{2}^{2 d-4} x^{2 d-5}}{(d-2)}= & \frac{(d-3)}{4 \pi} r_{2}^{2 d-6} x^{2 d-6} \\
& -\frac{q^{2}(d-3)}{4 \pi} \frac{\left(1-x^{2 d-5}\right)}{1-x}, \\
2 T_{0} r_{2}^{2 d-5} x^{2 d-5}= & 4 \operatorname{Pr}_{2}^{2 d-4} x^{2 d-5} \frac{1+x}{(d-2)} \\
& +\frac{(d-3)}{4 \pi} r_{2}^{2 d-6} x^{2 d-6}(1+x) \\
& -\frac{q^{2}(d-3)}{4 \pi}\left(1+x^{2 d-5}\right) .
\end{aligned}
$$

From (28) and (31), we can obtain

$$
\begin{gathered}
\frac{4 P r_{2}^{2 d-4} x^{2 d-5}}{(d-2)(d-1)}\left[(d-1)(1+x)\left(1-x^{d-2}\right)\right. \\
\left.-2(d-2)\left(1-x^{d-1}\right)\right] \\
+\frac{r_{2}^{2 d-6} x^{2 d-6}}{4 \pi}\left[(d-3)(1+x)\left(1-x^{d-2}\right)\right. \\
\left.\quad-2(d-2) x\left(1-x^{d-3}\right)\right] \\
-\frac{q^{2}}{4 \pi}\left[(d-3)\left(1+x^{2 d-5}\right)\left(1-x^{d-2}\right)\right. \\
\left.-2(d-2) x^{d-2}\left(1-x^{d-3}\right)\right]=0 .
\end{gathered}
$$

Substituting (30) into (32), one can derive

$$
r_{2}^{2(d-3)}=q^{2} \frac{y_{1}(x)}{y_{2}(x)}
$$

with

$$
\begin{aligned}
y_{2}(x)=2 x^{2 d-6} & \cdot\left[(d-3)(1+x)\left(1-x^{d-2}\right)\right. \\
& -\frac{(d-2)(d-3)}{(d-1)}\left(1-x^{d-1}\right) \\
& \left.-(d-2) x\left(1-x^{d-3}\right)\right], \\
y_{1}(x)=(d-3)(1+x)\left(1-x^{d-2}\right) \frac{\left(1-x^{2 d-5}\right)}{1-x} & (d-2)(d-3) \\
& -2 \frac{(d-1)}{\left(1-x^{d-1}\right) \frac{\left(1-x^{2 d-5}\right)}{1-x}} \\
+ & (d-3)\left(1+x^{2 d-5}\right)\left(1-x^{d-2}\right) \\
& -2(d-2) x^{d-2}\left(1-x^{d-3}\right) .
\end{aligned}
$$

Substituting (33) into (30), we have

$$
\begin{aligned}
& 4 P \frac{x^{2 d-5}}{(d-2)}\left(q^{2} \frac{y_{1}(x)}{y_{2}(x)}\right)^{(d-2) /(d-3)} \\
& \quad=q^{2} \frac{(d-3)}{4 \pi}\left[\frac{y_{1}(x)}{y_{2}(x)} x^{2 d-6}-\frac{\left(1-x^{2 d-5}\right)}{1-x}\right] .
\end{aligned}
$$

According to (33), when $x=1$,

$$
r_{c}^{2(d-3)}=q^{2} \lim _{x \rightarrow 1} \frac{y_{1}(x)}{y_{2}(x)} .
$$

According to $r_{c}$, we can derive from (30) the critical pressure

$$
P_{c}=\frac{(d-3)}{16 \pi r_{c}^{2}}
$$

Setting $P=\chi P_{c}$ with $\chi \leq 1$, (35) turns into

$$
\begin{aligned}
& \chi \frac{(d-3) x^{2 d-5}}{(d-2) r_{c}^{2}}\left(q^{2} \frac{y_{1}(x)}{y_{2}(x)}\right)^{(d-2) /(d-3)} \\
& \quad=q^{2}(d-3)\left[\frac{y_{1}(x)}{y_{2}(x)} x^{2 d-6}-\frac{\left(1-x^{2 d-5}\right)}{1-x}\right]
\end{aligned}
$$

In Table 2, we give the numerical values of $x, r_{1}, r_{2}$ and $T_{0}$ at different pressures and different spacetime dimensions.

\section{Latent Heat}

As is known, for RN-AdS black hole there is similar $P-V$ criticality to the Van der Waals liquid/gas system. This kind 
TABLE 1: Numerical solutions for $x, r_{1}, r_{2}$, and $P_{0}$ and the latent heat $L$ at different temperatures and different spacetime dimensions with $q=1$.

\begin{tabular}{|c|c|c|c|c|c|c|}
\hline$d$ & $\chi$ & $x$ & $r_{1}$ & $r_{2}$ & $P_{0}$ & $L$ \\
\hline \multirow{3}{*}{4} & 1 & 1 & 2.44949 & 2.44949 & 0.00331573 & 0 \\
\hline & 0.8 & 0.272204 & 1.47068 & 5.40288 & 0.00189057 & 2.94246 \\
\hline & 0.7 & 0.195886 & 1.34978 & 6.89065 & 0.00137985 & 4.34939 \\
\hline \multirow{3}{*}{5} & 1 & 1 & 1.96799 & 1.96799 & 0.0205468 & 0 \\
\hline & 0.8 & 0.361841 & 1.33342 & 3.68509 & 0.0123256 & 24.3525 \\
\hline & 0.7 & 0.277861 & 1.25038 & 4.50001 & 0.00921581 & 39.8572 \\
\hline \multirow{3}{*}{6} & 1 & 1 & 1.74258 & 1.74258 & 0.0589640 & 0 \\
\hline & 0.8 & 0.420776 & 1.26150 & 2.99802 & 0.0361453 & 96.7400 \\
\hline & 0.7 & 0.334087 & 1.19735 & 3.58395 & 0.0272937 & 176.243 \\
\hline
\end{tabular}

TABLE 2: Numerical solutions for $x, r_{1}, r_{2}$, and $T_{0}$ at different pressures and different spacetime dimensions with $q=1$.

\begin{tabular}{cccccc}
\hline$d$ & $\chi$ & $x$ & $r_{1}$ & $r_{2}$ \\
\hline 4 & 1 & 1 & 2.44949 & 2.44949 & 0.0433136 \\
& 0.8 & 0.441089 & 1.72015 & 3.89978 & 0.0397529 \\
5 & 0.6 & 0.289296 & 1.49696 & 5.17448 & 0.0353931 \\
\hline & 1 & 1 & 1.96799 & 1.96799 & 0.102438 \\
& 0.8 & 0.253434 & 1.22649 & 4.83950 & 0.0858594 \\
6 & 0.6 & 0.204914 & 1.17961 & 5.75663 & 0.0749342 \\
& 1 & 1 & 1.74258 & 1.74258 & 0.166356 \\
& 0.8 & 0.230015 & 1.12609 & 4.89572 & 0.125739 \\
\hline
\end{tabular}

of phase transition from liquid phase to gas phase is the firstorder phase transition. Therefore, in the process, there exists latent heat. The latent heat $L$ between the two phases $\alpha$ and $\beta$ is the difference between their molar enthalpies:

$$
L=H^{\beta}-H^{\alpha}=T\left(S^{\beta}-S^{\alpha}\right) .
$$

Here enthalpy is a thermodynamic function of temperature and pressure. The latent heat will change with the temperature

$$
\begin{aligned}
\frac{d L}{d T}= & \left(\frac{\partial H^{\beta}}{\partial T}\right)_{P}+\left(\frac{\partial H^{\beta}}{\partial P}\right)_{T} \frac{d P}{d T}-\left(\frac{\partial H^{\alpha}}{\partial T}\right)_{P} \\
& -\left(\frac{\partial H^{\alpha}}{\partial P}\right)_{T} \frac{d P}{d T} .
\end{aligned}
$$

Due to $C_{P}=(\partial H / \partial T)_{P}$ and $(\partial H / \partial P)_{T}=V-T(\partial V / \partial T)_{P}$, (40) becomes

$$
\begin{aligned}
\frac{d L}{d T}= & C_{P}^{\beta}-C_{P}^{\alpha}+\frac{L}{T} \\
& -\left[\left(\frac{\partial V^{\beta}}{\partial T}\right)_{P}-\left(\frac{\partial V^{\alpha}}{\partial T}\right)_{P}\right] \frac{L}{V^{\beta}-V^{\alpha}}
\end{aligned}
$$

The Clausius-Clapeyron equation is

$$
\left.\frac{d P}{d T}\right|_{\text {coexistence }}=\frac{s^{\beta}-s^{\alpha}}{v^{\beta}-v^{\alpha}}=\frac{S^{\beta}-S^{\alpha}}{V^{\beta}-V^{\alpha}},
$$

where $s^{\beta}, s^{\alpha}$ and $v^{\beta}, v^{\alpha}$ stand for the specific entropy and specific volume of the $\beta, \alpha$ phase, respectively. For the $\mathrm{RN}$ AdS black hole, from the equation of state we can calculate

$$
\begin{aligned}
& C_{P}=\left(\frac{\partial S}{\partial T}\right)_{P}=T\left(\frac{\partial S}{\partial r_{+}}\right)_{P}\left(\frac{\partial r_{+}}{\partial T}\right)_{P} \\
&=(d-2) S \\
& \cdot\left(\left(16 \pi P r_{+}^{2 d-4}+(d-2)(d-3) r_{+}^{2 d-6}\right.\right. \\
&\left.-q^{2}(d-2)(d-3)\right) \\
& \cdot\left(16 \pi P r_{+}^{2 d-4}-(d-2)(d-3) r_{+}^{2 d-6}\right. \\
&\left.\left.+q^{2}(d-2)(d-3)(2 d-5)\right)^{-1}\right) \\
&= T S\left(\left(4 \pi(d-2)^{2} r_{+}^{2 d-5}\right)\right. \\
& \cdot\left(16 \pi P r_{+}^{2 d-4}-(d-2)(d-3) r_{+}^{2 d-6}\right. \\
&\left.\left.+q^{2}(d-2)(d-3)(2 d-5)\right)^{-1}\right), \\
&\left(\frac{\partial V}{\partial T}\right)_{P}=V\left(\left(4 \pi(d-2)(d-1) r_{+}^{2 d-5}\right)\right. \\
& \quad\left(16 \pi r_{+}^{2 d-4}-(d-2)(d-3) r_{+}^{2 d-6}\right. \\
&\left.\left.+q^{2}(d-2)(d-3)(2 d-5)\right)^{-1}\right) .
\end{aligned}
$$




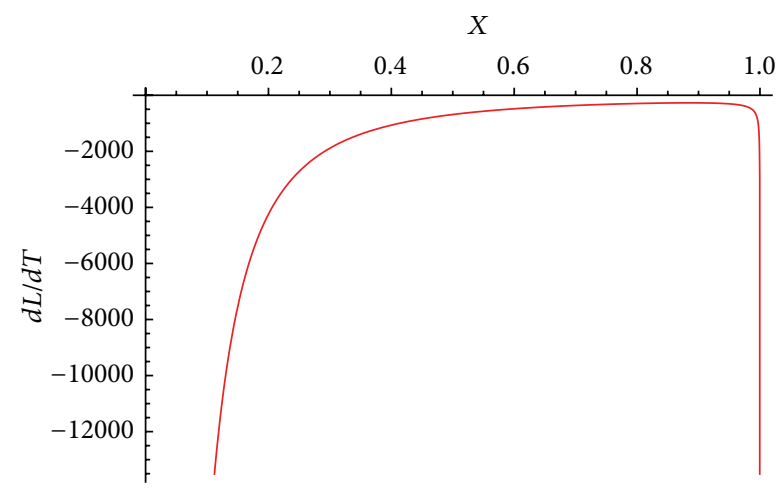

FIgURE 1: Plots of $d L / d T$ versus the parameter $\chi$ for the RN-AdS black hole. We have set $q=1$ and $d=4$.

Taking different temperatures, we can obtain different $x$, thus corresponding $r_{1}, r_{2}$. Set $r_{1}$ to be the $\alpha$ phase and $r_{2}$ to be the $\beta$ phase. We can calculate the latent heat $L$ at different temperatures and different spacetime dimensions. The result is shown in Table 1.

We can also depict the $d L / d T$ - $\chi$ curve which is shown in Figure 1 and can describe how the latent heat changes with the temperature.

\section{Conclusion}

We studied the thermodynamic behavior of $d$-dimensional $\mathrm{RN}$-AdS black hole in the extended phase space. If the black holes really express themselves like Van der Waals liquidgas system, in the isotherms of the AdS black holes there must be regimes where the condition of stable equilibrium is violated. Analogous to the VdW system the unphysical oscillating part in the isotherm should be replaced with an isobar, which represents a coexistence line. According to the equal area law, we find out the position of the isobars in the $P-V$ plane at different temperatures $T<T_{c}$ and the isobars in the $T$-S plane at different pressures. It is shown that the length of the isobar indeed depends on the temperature and pressure. In the $P-V$ plane, the higher the temperature is, the shorter the isobar will be. The same case happens in the $T$-S plane. If the phase diagrams of the black holes are indeed the case, the evolution of black holes will be very different from the usual Hawking behavior during the coexistence regime, where the temperature and the pressure are both constant. Although the equal area law has been used to construct the phase diagram of the charged AdS black hole in the T-S plane in [29], the method we used is different. We start from the chemical potential or the Gibbs potential to obtain the equal area law. We derive the position of the coexistence isobar by introducing two functions $y_{1}(x), y_{2}(x)$. Taking the limit, $x \rightarrow 1$, one can easily obtain the critical position according to $y_{1}(x), y_{2}(x)$. Thus the other critical thermodynamic quantities can be calculated easily. For some complicated black holes, such as Gauss-Bonnet-AdS one, the method will be more effective to obtain the critical value of the thermodynamic quantities. In addition, we also discussed the latent heat $L$ for the RN-AdS black hole. It can be shown that $L$ will increase as the temperature decreases. Figure 1 shows the relation between $d L / d T$ and temperatures.

\section{Conflict of Interests}

The authors declare that there is no conflict of interests regarding the publication of this paper.

\section{Acknowledgments}

This work was supported in part by the National Natural Science Foundation of China (Grant nos. 11075098 and 11175109), the Young Scientists Fund of the National Natural Science Foundation of China (Grant no. 11205097), the Natural Science Foundation for Young Scientists of Shanxi Province, China (Grant no. 2012021003-4), and the Shanxi Datong University Doctoral Sustentation Fund (nos. 2008-B06 and 2011-B-04), China.

\section{References}

[1] J. M. Bardeen, B. Carter, and S. W. Hawking, "The four laws of black hole mechanics," Communications in Mathematical Physics, vol. 31, pp. 161-170, 1973.

[2] P. Davies, "The thermodynamic theory of black holes," Proceedings of the Royal Society of London A, vol. 353, no. 1675, pp. 499521, 1977.

[3] S. W. Hawking and D. N. Page, "Thermodynamics of black holes in anti-de Sitter space," Communications in Mathematical Physics, vol. 87, no. 4, pp. 577-588, 1983.

[4] P. Hut, "Charged black holes and phase transitions," Monthly Notices of the Royal Astronomical Society, vol. 180, no. 3, pp. 379389, 1977.

[5] L. M. Sokolowski and P. Mazur, "Second-order phase transitions in black-hole thermodynamics," Journal of Physics A, vol. 13, no. 3, pp. 1113-1120, 1980.

[6] C. O. Lousto, "Emergence of an effective two-dimensional quantum description from the study of critical phenomena in black holes," Physical Review D, vol. 51, no. 4, pp. 1733-1740, 1995.

[7] C. S. Peça and J. P. S. Lemos, "Thermodynamics of ReissnerNordström-anti-de Sitter black holes in the grand canonical ensemble," Physical Review D, vol. 59, no. 12, Article ID 124007, 1999.

[8] R. Banerjee, S. K. Modak, and S. Samanta, "Second order phase transition and thermodynamic geometry in Kerr-AdS black holes," Physical Review D, vol. 84, Article ID 064024, 2011.

[9] R. Banerjee and D. Roychowdhury, "Critical phenomena in born-infeld AdS black holes," Physical Review D, vol. 85, Article ID 044040, 2012.

[10] Y. S. Myung, "Phase transitions for Lifshitz black holes," The European Physical Journal C, vol. 72, article 2116, 2012.

[11] A. Chamblin, R. Emparan, C. V. Johnson, and R. C. Myers, "Charged AdS black holes and catastrophic holography," Physical Review D, vol. 60, no. 6, 1999.

[12] A. Chamblin, R. Emparan, C. V. Johnson, and R. C. Myers, "Holography, thermodynamics, and fluctuations of charged AdS black holes," Physical Review D, vol. 60, no. 10, Article ID 104026, 20 pages, 1999. 
[13] X. N. Wu, "Multicritical phenomena of Reissner-Nordström anti-de Sitter black holes," Physical Review D, vol. 62, no. 12, Article ID 124023, 2000.

[14] D. Kastor, S. Ray, and J. Traschen, "Enthalpy and the mechanics of AdS black holes," Classical and Quantum Gravity, vol. 26, no. 19, Article ID 195011, 16 pages, 2009.

[15] M. Cvetic, G. Gibbons, D. Kubiznak, and C. Pope, "Black hole enthalpy and an entropy inequality for the thermodynamic volume," Physical Review D, vol. 84, Article ID 024037, 2011.

[16] C. Niu, Y. Tian, and X. N. Wu, "Critical phenomena and thermodynamic geometry of Reissner-Nordström-anti-de Sitter black holes," Physical Review D, vol. 85, no. 2, Article ID 024017, 8 pages, 2012.

[17] R. Banerjee, S. K. Modak, and D. Roychowdhury, "A unified picture of phase transition: from liquid-vapour systems to AdS black holes," Journal of High Energy Physics (JHEP), vol. 1210, p. 125, 2012.

[18] S.-W. Wei and Y.-X. Liu, "Critical phenomena and thermodynamic geometry of charged Gauss-Bonnet AdS black holes," Physical Review D, vol. 87, Article ID 044014, 2013.

[19] M. B. Jahani Poshteh, B. Mirza, and Z. Sherkatghanad, "Phase transition, critical behavior, and critical exponents of MyersPerry black holes," Physical Review D-Particles, Fields, Gravitation and Cosmology, vol. 88, no. 2, Article ID 024005, 2013.

[20] R. Zhao, M. Ma, H. Li, and L. Zhang, "On thermodynamics of charged and rotating asymptotically AdS black strings," Advances in High Energy Physics, vol. 2013, Article ID 371084, 7 pages, 2013.

[21] R. Zhao, H. H. Zhao, M. S. Ma, and L. C. Zhang, "On the critical phenomena and thermodynamics of charged topological dilaton AdS black holes," European Physical Journal C, vol. 73, no. 12, pp. 1-10, 2013.

[22] M.-S. Ma, H.-H. Zhao, L.-C. Zhang, and R. Zhao, "Existence condition and phase transition of Reissner-Nordström-de Sitter black hole," International Journal of Modern Physics A, vol. 29, no. 9, Article ID 1450050, 2014.

[23] R. Zhao, M.-S. Ma, H.-H. Zhao, and L.-C. Zhang, "The critical phenomena and thermodynamics of the Reissner-Nordstromde Sitter black hole," Advances in High Energy Physics, vol. 2014, Article ID 124854, 6 pages, 2014.

[24] B. P. Dolan, "Pressure and volume in the first law of black hole thermodynamics," Classical and Quantum Gravity, vol. 28, no. 23, Article ID 235017, 2011.

[25] D. Kubiznak and R. B. Mann, "P-V criticality of charged AdS black holes," Journal of High Energy Physics, vol. 2012, no. 7, article 033, 2012.

[26] S. Gunasekaran, D. Kubiznak, and R. B. Mann, "Extended phase space thermodynamics for charged and rotating black holes and Born-Infeld vacuum polarization," Journal of High Energy Physics, no. 11, pp. 1-43, 2012.

[27] R.-G. Cai, L.-M. Cao, and R.-Q. Yang, "PV criticality in the extended phase space of Gauss-Bonnet black holes in AdS space," Journal of High Energy Physics, vol. 2013, no. 9, article 5, 2013.

[28] S. H. Hendi and M. H. Vahidinia, "Extended phase space thermodynamics and P-V criticality of black holes with a nonlinear source," Physical Review D, vol. 88, no. 8, Article ID 084045, 11 pages, 2013.

[29] E. Spallucci and A. Smailagic, "Maxwell's equal-area law for charged Anti-de Sitter black holes," Physics Letters. B, vol. 723, no. 4-5, pp. 436-441, 2013. 

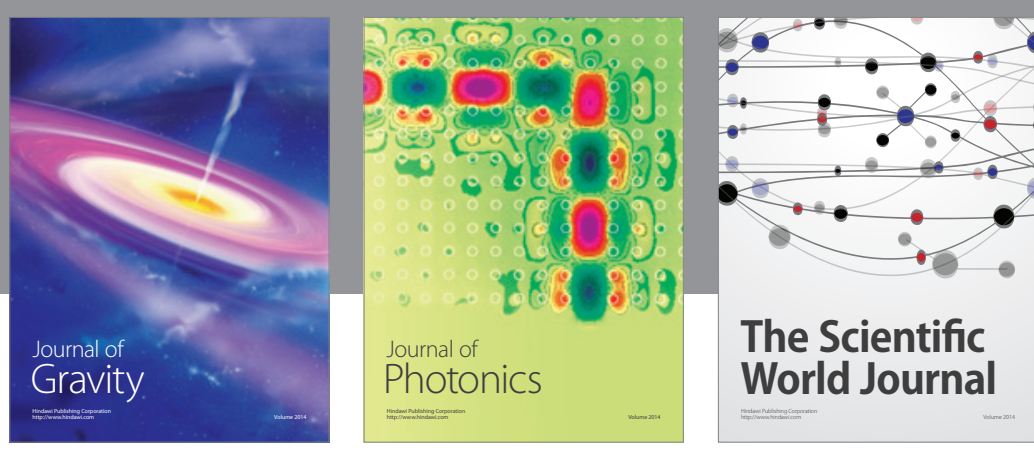

The Scientific World Journal
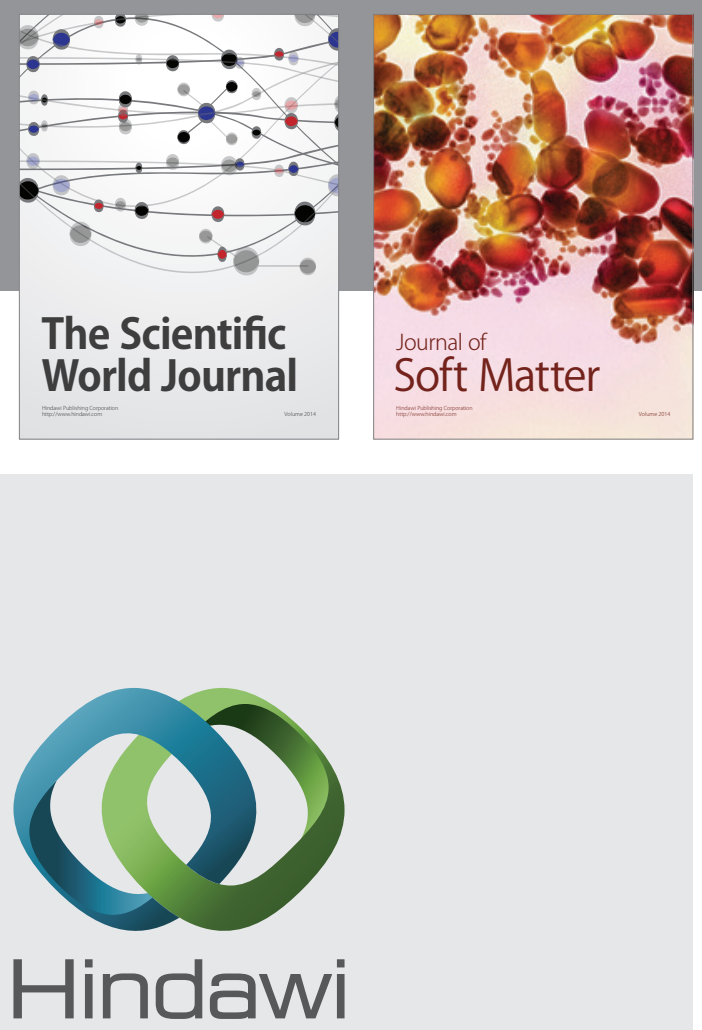

Submit your manuscripts at

http://www.hindawi.com

nternational Journal of

Statistical Mechanics
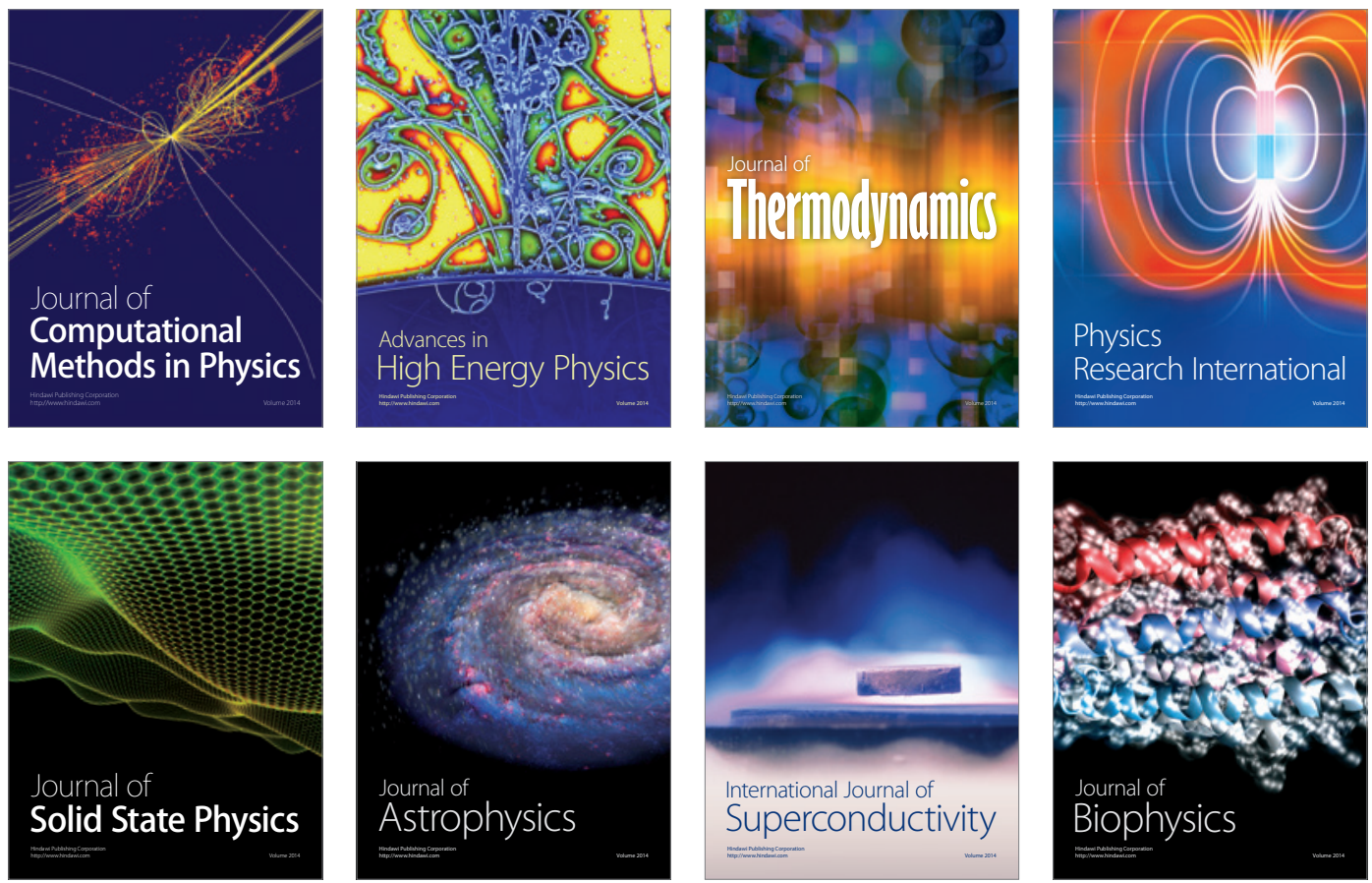
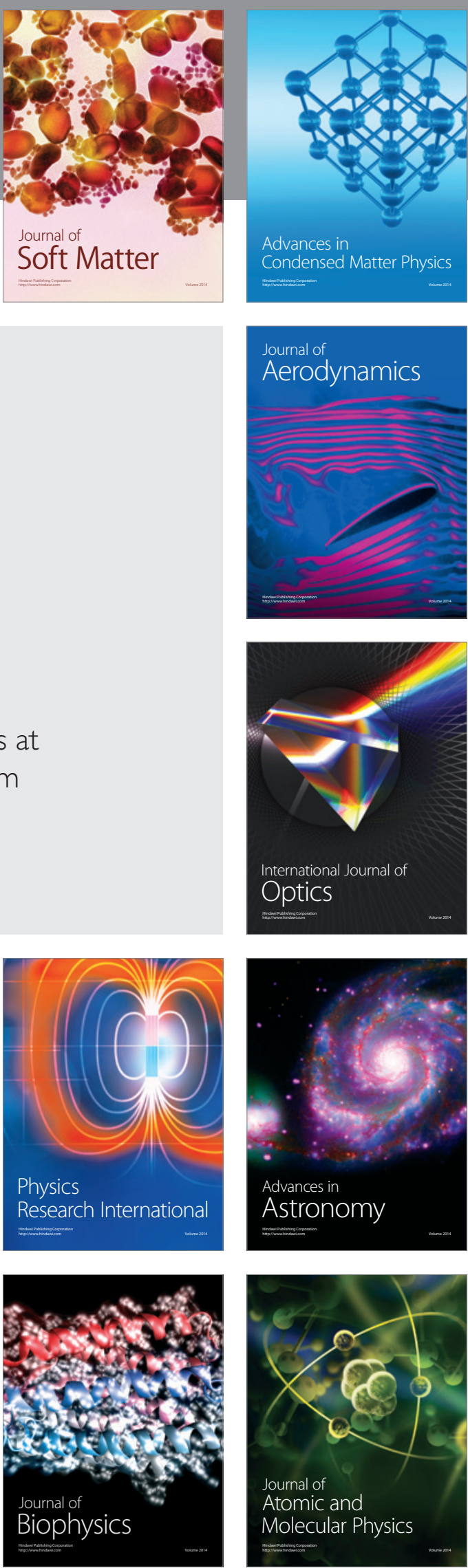